\title{
Il Rene Policistico e l'A.I.R.P. onlus
}

\section{Rene policistico autosomico dominante: nuovi aspetti terapeutici}

\section{R. Magistroni}

Divisione di Nefrologia Dialisi e Trapianto, Dipartimento di Medicina e Specialità Mediche, Azienda Ospedaliero Universitaria Policlinico di Modena, Università di Modena e Reggio Emilia

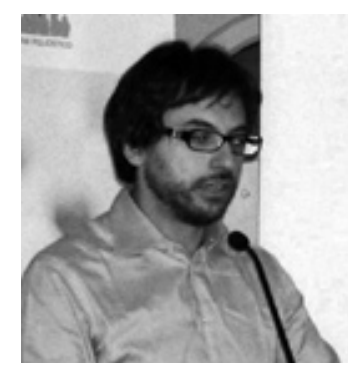

\section{Introduzione}

Il rene policistico autosomico dominante $(\mathrm{ADPKD})$ è una patologia genetica a carattere mendeliano semplice. La patogenesi della malattia deriva dalla alterazione genetica di uno dei due geni coinvolti: PKD1, PKD2. La circoscrizione delle cause prime della patologia a un unico gene e quindi a un'unica proteina e infine a un'unica funzione cellulare promuove le patologie monogeniche a modelli patogenetici di particolare interesse. Infatti questa peculiare condizione in cui a un'unica e definita causa iniziale è attribuibile la successiva cascata di alterazioni patofisiologiche si configura come un grimaldello capace di scardinare e mettere in evidenza sconosciuti meccanismi cellulari che possono essere finalmente compresi. Per questo motivo le malattie monogeniche hanno costituito nell'ultimo decennio i modelli patologici umani su cui la ricerca scientifica ha ottenuto il maggiore vantaggio conoscitivo. $\mathrm{ADPKD}$ non fa eccezione: l'esplosione di letteratura scientifica su questo argomento ha moltiplicato il livello di conoscenza. Il corpus di dati acquisiti si è coagulato nella teoria del ciglio primario, cuore teorico comune alla patogenesi di diverse malattie cistiche renali. Infine la conoscenza delle cause ha suggerito interventi terapeutici fino a pochi anni fa neppure ipotizzabili.

Da un punto di vista strategico l'evoluzione di ADPKD può essere scomposta in tre fasi successive ciascuna delle quali suggerisce strategie di intervento diverse: a) alterazione genetica - mutazione di PKD1 o PKD2; b) comparsa ed espansione delle cisti; c) progressione del danno. Per quanto riguarda le strategie correttive della alterazione genetica almeno due possibili interventi potrebbero essere ipotizzati. Il primo potrebbe essere la correzione dell'errore genetico che in ultima analisi è il primo evento causativo della patologia. Il secondo riguarda la riparazione del tessuto geneticamente alterato attraverso la sua sostituzione con un tessuto sano rigenerato attraverso la differenziazione di cellule staminali. Entrambi questi approcci sono al momento tecnologicamente immaturi, non sono disponibili trial clinici che impieghino queste strategie e per questo motivo non ne tratteremo in questa revisione.

Molto più attivo è invece il campo riguardante le strategie di rallentamento dei fenomeni di crescita delle cisti. Questo gruppo di strategie è il più rappresentato nei termini di protocolli clinici attivi. Consultando il sito "ClinicalTrials.gov" mantenuto dall"'U.S. National Institutes of Health" (NIH) che permette la ricerca all'interno di un database di trial clinici registrati si possono attualmente conteggiare 28 trial inerenti ADPKD. Di questi il $17 \%$ è costituito da trial di fase II/III mentre il $39 \%$ da trial di fase III (Fig. 1). Come vedremo gli interventi terapeutici riguardano molecole che sviluppate in altri contesti e con altre indicazioni cliniche si sono dimostrate attive nelle vie cellulari alterate in ADPKD. In questo commento ridurremo la discussione a due categorie di molecole, gli inibitori di mTOR e gli analoghi biologici della somatostatina poiché solo per queste due categorie sono stati conclusi e pubblicati trial clinici di fase III.

Infine la terza strategia riguarda tecnologie dirette alla riduzione dei fenomeni di progressione del danno renale. Queste strategie impiegano farmaci non specifici per $\mathrm{ADPKD}$ ma più genericamente indicati in tutte le condizioni in cui si è instaurata una condizione di insufficienza renale cronica. Tra questi farmaci rientrano ad esempio le molecole attive sul sistema renina-angiotensina. Pur promettendo importanti avanzamenti tera- 


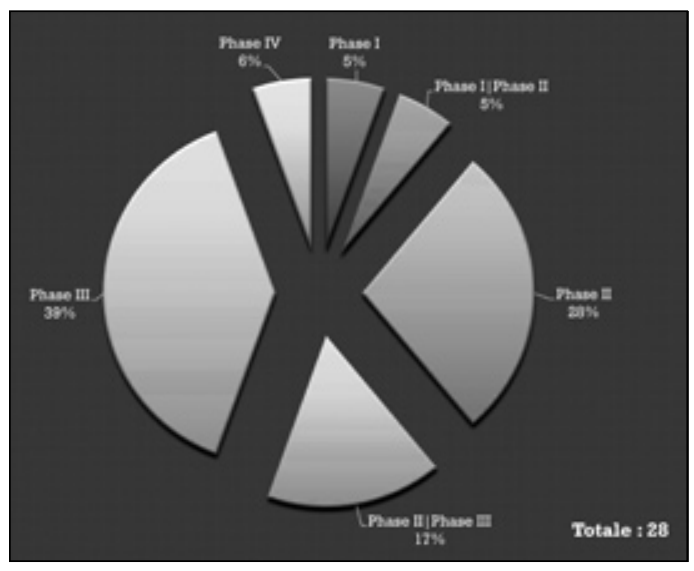

Fig. 1 - Trial clinici attivi di fase I - II - III e IV in ADPKD. Dati ottenuti dalla fonte ClinicalTrials.gov.

peutici per la loro indicazione generale non verranno trattati in questa revisione.

\section{Gli inibitori di mTOR}

\section{I modelli animali}

La proteina target della rapamicina (mTOR) è una serin-treonin chinasi conservata in senso evolutivo che costituisce il core catalitico di due complessi proteici (mTORC1 e mTORC2). La somministrazione di rapamicina, o del derivato everolimus, agiscono inibendo l'assemblaggio del complesso mTORC1, e limitatamente ad alcuni tessuti in somministrazione cronica possono inibire l'assemblaggio di mTORC2. mTORC1 è un sensore di specifici segnali intra ed extra cellulari che promuove processi anabolici e inibisce processi catabolici. Fattori di crescita e nutrienti (aminoacidi, substrati energetici ecc.) promuovono la sintesi proteica mTORC1 dipendente, crescita cellulare (nel senso di un aumento della massa cellulare) e il metabolismo cellulare. Al contrario una riduzione di segnale di $\mathrm{mTORC} 1$ riduce la biosintesi cellulare e promuove processi di autofagia coerentemente alla condizione di status sub ottimale della cellula (1). $\grave{E}$ stato dimostrato che la coda citoplasmatica della policistina-1 (la proteina codificata dal gene PKD1) interagisce con la proteina tuberina, un fattore di regolazione di mTOR. Conseguentemente mTOR risulta inappropriatamente attivato nelle cellule epiteliali che tappezzano le cisti renali in pazienti ADPKD e in modelli murini(2). Questi elementi hanno suggerito un possibile ruolo terapeutico degli inibitori di mTOR in ADPKD nellipotesi che questa classe di farmaci possa inibire i fenomeni proliferativi che sono alla base dell'alterazione cistica. Gli inibitori di mTOR sono stati testati negli anni 20052009 su alcuni animali con fenotipo cistico renale (ratto Han:SPRD (3-7), topo orpk, topo bpk $(8,9))$ ottenendo risultati incoraggianti. Questi modelli pur presentando un fenotipo renale cistico non costituiscono modelli geneticamente singenici rispetto alla patologia umana. Questa lacuna sperimentale è stata recentemente colmata impiegando un modello murino nel quale si è ottenuta una inattivazione condizionale di PKD1 limitata al rene. Anche in questo caso è stato confermato un effetto benefico della rapamicina (2).

Oltre alla proprietà antiproliferativa anche una ipotesi anti angiogenetica è stata proposta come effetto terapeutico degli inibitori di mTOR. È notorio che ADPKD presenta un rimodellamento dell'albero vascolare renale e vi sono elementi che suggeriscono un fenomeno neoangiogenetico in prossimità delle cisti renali (10).

Le proprietà anti neoangiogenetiche degli inibitori di mTOR sono note e gli si attribuiscono parte del potenziale antitumorale riconosciuto a questa classe di farmaci $(11,12)$.

\section{I trial clinici}

La disponibilità di dati preclinici così incoraggianti ha ispirato il disegno di diversi trial clinici. Al momento sono disponibili i dati definitivi di tre studi di cui uno con caratteristiche di studio pilota(13) e altri due con numerosità più significativa $(14,15)$. I dati di questi due ultimi studi erano attesi con grande ansia dalla comunità scientifica: le alte aspettative si sono purtroppo infrante contro l'evidenza di risultati non propriamente incoraggianti. Tutti e tre gli studi non hanno potuto dimostrare un effetto positivo del trattamento (Tab. I). Lo studio SIRENA è uno studio pilota che ha reclutato 21 pazienti, pur avendo dimostrato alcuni dati interessanti nelle sottoanalisi morfologiche il suo significato è stato complessivamente soppiantato dai due studi più recenti che hanno numerosità più significativa.

Lo "Suisse Trial"(14) è uno studio "open label" randomizzato controllato che ha reclutato 100 pazienti di età compresa tra 18 e 40 anni. I pazienti arruolati nel braccio sperimentale hanno assunto $2 \mathrm{mg} /$ die di Sirolimus. Il reclutamento prevedeva un filtrato glomerulare superiore o uguale a $70 \mathrm{ml} / \mathrm{min}$ e l'evidenza di un incremento volumetrico renale superiore al $2 \%$ in un periodo di 6 mesi. L' outcome primario come nella maggior parte degli studi condotti fino a questo momento prevedeva la valutazione delle variazioni volumetriche dei reni. La variazione del volume renale in vece della variazione degli indici di funzione renale è un classico esempio di outcome cli- 
nico surrogato la cui validità è stata precedentemente suggellata dalle esperienze del "Consortium for Radiologic Imaging Studies of Polycystic Kidney Disease" (CRISP) (16). Proprio questi trial come commenterò in seguito hanno parzialmente messo in crisi l'accettabilità del volume renale come outcome surrogato. Il trial non ha potuto dimostrare alcuna differenza tra i due gruppi sperimentali. Peraltro i due gruppi di pazienti hanno manifestato nei 18 mesi di follow-up incrementi volumetrici relativamente modesti e ampia variabilità. Questa condizione pone dubbi sulla reale potenza di questo studio: la dimostrazione di una qualsiasi efficacia avrebbe verosilmente richiesto o una attività farmacologica straordinariamente elevata ovvero lo studio avrebbe dovuto reclutare un numero di pazienti assai superiore.

"Everolimus Trial" (15) è un protocollo randomizzato in doppio cieco che ha testato l'efficacia della molecola Everolimus al dosaggio di 2,5 $\mathrm{mg}$ due volte al giorno rispetto a placebo. Ha reclutato 433 pazienti in 24 Centri partecipanti e i soggetti sono stati seguiti per 24 mesi. Anche questo studio ha fallito nel dimostrare una differenza significativa nella variazione volumetrica dei reni nei due gruppi così come non ha potuto dimostrare un vantaggio nella preservazione della funzione renale.
Piuttosto la valutazione dei dati suggerisce al contrario un possibile peggioramento funzionale del gruppo sperimentale. I dati ottenuti da questo studio sono in forte controtendenza rispetto ai precedenti dati preclinici.

Prima di trarre ogni conclusione definitiva credo valga la pena di valutare alcuni elementi critici di questo studio (Tab. I). A suo favore va certamente attribuito la numerosità del campione reclutato (433 pazienti) che certamente pone questo studio al di sopra dei precedenti. È necessario però sottolinearne le principali e non trascurabili debolezze. Si è registrato durante il follow-up un assai significativo drop out maggiormente concentrato nel gruppo sperimentale che ha fortemente condizionato la potenza statistica dello studio. Il fatto che i drop out si siano concentrati nel gruppo sperimentale solleva il dubbio che i pazienti in trattamento con everolimus abbiano sofferto di un significativo numero di eventi avversi legati al farmaco. In questo trial infatti è stato utilizzato il dosaggio più elevato (everolimus 2,5 $\mathrm{mg} \mathrm{x} \mathrm{2/die)}$ rispetto agli altri due studi pubblicati. La conduzione dello studio è stata sub ottimale per un elevatissimo numero di violazioni di protocollo: le violazioni hanno principalmente riguardato la mancata disponibilità di esami di risonanza magnetica con cui veniva stimato il volume

TABELLA I - TABELLA RIASSUNTIVA DI COMPARAZIONE DELLE PRINCIPALI CARATTERISTICHE DEI TRE TRIAL CLINICI PUBBLICATI SULLUTILIZZO DI INIBITORI DI mTOR
$\operatorname{SIRENA}_{(13)}$
SUISSE (14)
EVEROLIMUS (15)

\begin{tabular}{llll}
\hline Pazienti & $21(15)$ & 100 & $433(346)$
\end{tabular}

Terapia Sper. Sirolimus Sirolimus $\quad$ Everolimus

$\begin{array}{llll} & 3 \mathrm{mg} / \mathrm{die} & 2 \mathrm{mg} / \mathrm{die} & 2,5 \mathrm{mg} \times 2 / \mathrm{die} \\ \text { Dosaggio } & (5-10 \mathrm{ng} / \mathrm{ml}) & (4-10 \mathrm{ng} / \mathrm{ml}) & (3-8 \mathrm{ng} / \mathrm{ml})\end{array}$
GFR
$76.5 \pm 28.3$
$92 \pm 20$
$53 \pm 19.8$

\begin{tabular}{llll} 
Volume renale & $1874 \pm 1057$ & $907(577-1330)$ & $2028 \pm 1173$ \\
\hline Outcome & Volume renale (CT) & Volume Renale(MRI) & Volume Renale (MRI) \\
Efficace & (No) & No & No \\
\hline Punto di forza & Analisi di immagini & Basso drop out & (Numerosità) \\
\hline Limiti & Studio Pilota & $\begin{array}{l}\text { Arruolam. Limitato } \\
\text { Ampia variabilita della popolazione } \\
\text { Follow up limitato }\end{array}$ & $\begin{array}{l}\text { Alto Drop out } \\
\text { Violaz. Protocollo } \\
\text { Follow up limitato }\end{array}$ \\
\hline
\end{tabular}




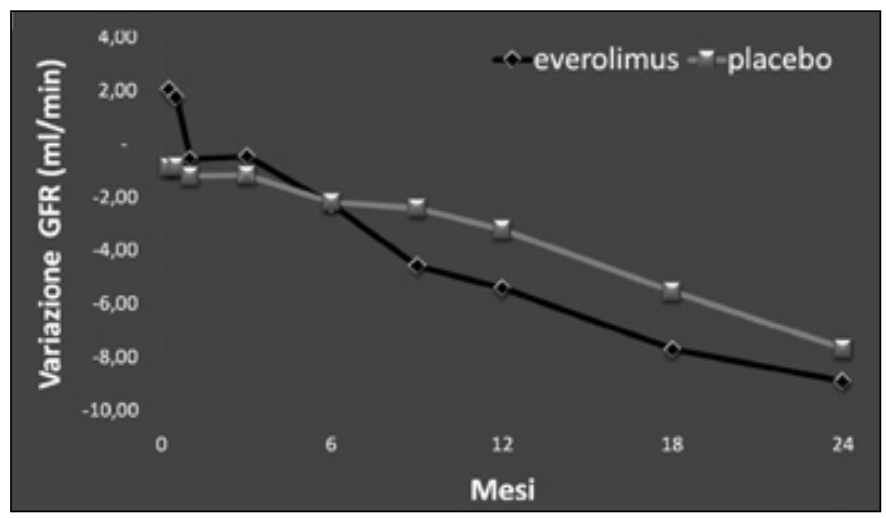

Fig. 2 - Variazione del filtrato glomerulare rispetto alla registrazione basale nei pazienti in trattamento con everolimus e nei pazienti in trattamento placebo (15).

renale, anche in questo caso adottato come outcome primario. I dati dopo un anno di follow-up dimostravano una significativa differenza di volume renale nei due gruppi favorevole per il gruppo sperimentale (volume renale inferiore nel gruppo trattato con everolimus); questa differenza statistica veniva meno alla fine del secondo anno di follow-up. Come anticipato questo dato negativo potrebbe essere stato causato dall'elevata violazione di protocollo con relativa perdita di potenza statistica. Il dato più allarmante riguarda però la registrazione di una tendenza a un più rapido declino della funzione renale nel gruppo in trattamento sperimentale. In autorevoli editoriali pubblicati a commento di questo articolo diverse ipotesi sono state proposte. In particolare è stato criticato che la terapia con everolimus avrebbe potuto ridurre le capacità rigenerative soprattutto riducendo la sopravvivenza endoteliale (attività anti neoangiogenetica) e riducendo l'integrità podocitaria (17).

Ulteriormente si è commentato che everolimus ha un effetto di contrasto ai fenomeni di iperfiltrazione glomerulare. Sulla base di questo meccanismo la riduzione del grado di filtrazione glomerulare avrebbe inizialmente determinato un apparente peggioramento funzionale. Ulteriormente si è ipotizzato che il corto follow-up non abbia permesso di cogliere gli effetti protettivi a lungo termine. In questo senso l'estrapolazione di alcuni dati non direttamente pubblicati ma disponibili nella versione on line della pubblicazione permette di produrre il grafico riprodotto nella Figura 2. Il grafico suggerirebbe effettivamente un significativo peggioramento funzionale a partire dal 6 mese che apparentemente tende a una stabilizzazione negli ultimi mesi di follow-up. Queste suggestioni potranno essere confermate unicamente dalla eventuale pubblicazione di dati di follow-up aggiuntivi.

\section{La somatostatina}

\section{Dati preclinici e modelli animali}

Il razionale dell'uso della somatostatina in $\mathrm{ADPKD}$ origina dalla evidenza della sua possibile azione di riduzione a livello tubulare dei livelli di AMPc. È noto infatti che le cellule tubulari renali presentano recettori per la somatostatina (recettori sst2), la cui stimolazione induce una riduzione dei livelli intracellulari disponibili di AMPc. L'AMPc agisce nel tubulo in complesse vie cellulari che promuovono il trasporto attivo di cloro, processo che guida la secrezione di fluidi a livello tubulare e che in $\mathrm{ADPKD}$ è uno dei fenomeni causativi del progressivo ingrandimento delle cisti renali (18). È noto inoltre che AMPc funge da secondo messaggero nella stimolazione della proliferazione cellulare fenomeno a sua volta coinvolto nell'ingrandimento delle lesioni cistiche (19). Recettori della somatostatina sono presenti anche nei colangiociti e attraverso meccanismi analoghi (riduzione della secrezione di fluidi, inibizione della proliferazione) la somatostatina avrebbe effetti potenzialmente protettivi della complicanza cistica epatica. L'octreotide, un analogo della somatostatina, ha dimostrato di ridurre il peso e l'indice mitotico del fegato di modelli murini di ADPKD (19).

Infine sono stati descritti tre casi riguardanti l'uso continuativo di octreotide in pazienti ADPKD il cui trattamento era stato giustificato da una sottostante patologia endocrina per le quali il farmaco è attualmente registrato. In questi pazienti il trattamento aveva determinato una concomitante riduzione volumetrica di reni e fegato $(20,21)$.

\section{I trial clinici}

La convergenza dei dati preclinici a favore del possibile beneficio dell'utilizzo di questo farmaco in ADPKD ha ispirato alcuni trial clinici esplorativi. Il primo ad essere stato pubblicato è il "Safety and efficacy of long-acting somatostatin treatment in autosomal-dominant polycystic kidney disease” pubblicato nel 2005 da Ruggeneti et al (21). E uno studio pilota con disegno "cross over" che ha reclutato 14 pazienti con un follow-up di 6 mesi. I pazienti sono stati trattati con octreotide al dosaggio di $40 \mathrm{mg}$ (due iniezioni intraglutee da $20 \mathrm{mg}$ ) ogni 28 giorni per 6 mesi. Sono stati eclusi dal reclutamento i pazienti con litiasi biliare, noto fattore di rischio per complicanze colelitiasiche nel trattamento con somatostatina. Lo studio ha dimostrato in questo piccolo campione un effetto di 
riduzione del volume totale renale nei pazienti durante il trattamento con octreotide. In una sotto analisi gli autori hanno suggerito che sebbene si potesse registrare una riduzione di crescita delle cisti renali, l'apporto più significativo nel decremeto del volume renale era imputabile al parenchima renale che appariva complessivamente ridotto rispetto al basale. Nel 2010 è stata infine pubblicata un analisi post hoc su questo stesso trial focalizzata sulla valutazione degli effetti epatici (22). I dati mostrano una significativa riduzione del volume epatico rispetto al dato basale e anche in questo caso la quota di riduzione è attribuile in parte maggiore alla componente parenchimale rispetto al compartimento cistico. Dati concordanti sull'outcome epatico sono stati pubblicati negli ultimi due anni da parte di ulteriori due gruppi di ricerca $(23,24)$. Il lavoro pubblicato da van Keimpema et al ha utilizzato il Lanreotide, un analogo della somatostatina ma con una affinità inferiore per i recettori sst2 ripetto all'octreotide.

\section{Conclusioni}

La recente pubblicazione di trial clinici inerenti il trattamento specifico della progressione in senso cistico della malattia renale in ADPKD ha aperto una stagione nuova in questa patologia. Ha infatti appena avuto inizio un periodo estremamente eccitante sotto il profilo scientifico e clinico che permetterà la valutazione critica delle strategie terapeutiche che gli ultimi 20 anni di ricerca di base hanno proficuamente alimentato. Purtroppo il primo di questi appuntamenti ha avuto un epilogo quantomeno problematico. L'inibizione di mTOR, uno dei meccanismi più promettenti sotto il profilo terapeutico, ha fallito in senso stretto nella dimostrazione di efficacia. Una lettura critica e attenta dei due trial e in particolare di quello tedesco pone profondi interrogativi e allontana dalla facile conclusione di una definitiva mancanza di efficacia. In particolare sembra plausibile che il farmaco ottenga una riduzione volumetrica dei reni, ma diviene problematica la dicotomia tra relativa preservazione morfologica e perdita di funzione renale. Le posizioni a detrimento ipotizzano una riduzione delle capacità rigenerative come effetto collaterale della inibizione di mTOR. Una cauta posizione a difesa ipotizza un concomitante effetto di riduzione della iperfiltrazione glomerulare che avrebbe mascherato un fenomeno protettivo di cui si potrebbe palesare il beneficio in tempi successivi (una prospettiva che evoca immediatamente nella esperienza nefrologica paradigmi ben noti della nefroprotezione da farmaci antagonisti del sistema renina angiotensina). Oltre alla disputa di efficacia, questo trial pone un interrogativo metodologico che dovrà essere preso in considerazione nel disegno sperimentale dei trial a venire. Infatti lo studio di Walz et al compromette il paradigma centrale degli studi fin qui disegnati, ovvero l'accettazione del volume renale come outcome surrogato della funzione renale. Lo scollamento che si registra in questo studio tra andamento della funzione renale $\mathrm{e}$ volume renale mette in forte crisi questo assunto. Infatti pur rimanendo valida la relazione tra volume e funzione renale registrata negli studi del gruppo CRISP, non possiamo assumere che questa relazione sia mantenuta in un contesto biologico diverso in cui viene inserito nel modello un elemento nuovo (nello specifico un farmaco attivo nel rimodellamento tissutale) mai quantificato negli studi del gruppo CRISP (16).

Per quanto riguarda la somatostatina gli studi pubblicati mostrano in modo complessivamente coerente un effetto positivo su outcome surrogati e in particolare relativi alle dimensioni epatiche. Ciononostante l'applicazione clinica di questi risultati deve ancora essere valutata con estrema cautela. Nessuno dei farmaci analoghi della somatostatina è registrato per la sua applicazione in $\mathrm{ADPKD}$ e ogni impiego in questo senso è da ritenersi "off label". Il numero di pazienti ADPKD complessivamente trattati è ancora esiguo. Pur apparendo ben tollerata non è possibile definire ancora il profilo di sicurezza di questa categoria di farmaci nei pazienti ADPKD. Infine bisogna segnalare la tendenza degli analoghi della somatostatina ad accumularsi nei pazienti affetti da insufficienza renale e che nessuno degli studi riportati ha valutato il trattamento in pazienti affetti da insufficienza renale e tanto meno in trattamento sostitutivo.

\section{Indirizzo degli Autori:}

Riccardo Magistroni, MD

Divisione di Nefrologia Dialisi e Trapianto

Azienda Ospedaliero Universitaria

Policlinico di Modena

Via Del Pozzo 71

41100 Modena

Riccardo.Magistroni@unimore.it 


\section{Bibliografia}

1. Foster KG, Fingar DC. Mammalian target of rapamycin (mTOR): conducting the cellular signaling symphony. J Biol Chem 2010; 285(19): 14071-7. PMCID: 2863215.

2. Shillingford JM, Piontek KB, Germino GG, Weimbs T. Rapamycin ameliorates PKD resulting from conditional inactivation of Pkd1. J Am Soc Nephrol 2010; 21(3): 48997. PMCID: 2831854.

3. Tao Y, Kim J, Schrier RW, Edelstein CL. Rapamycin markedly slows disease progression in a rat model of polycystic kidney disease. J Am Soc Nephrol 2005; 16(1): 46-51.

4. Wahl PR, Serra AL, Le Hir M, Molle KD, Hall MN, Wuthrich RP. Inhibition of mTOR with sirolimus slows disease progression in Han: SPRD rats with autosomal dominant polycystic kidney disease (ADPKD). Nephrol Dial Transplant 2006. PMCID: 207.

5. Zhang T, Wang L, Xiong X, Mao Z, Mei C. Mycophenolate mofetil versus Rapamycin in Han: SPRD rats with Polycystic Kidney Disease. Biol Res 2009; 42(4): 437-44.

6. Wu M, Wahl PR, Le Hir M, Wackerle-Men Y, Wuthrich RP, Serra AL. Everolimus retards cyst growth and preserves kidney function in a rodent model for polycystic kidney disease. Kidney Blood Press Res 2007; 30(4): 253-9.

7. Zafar I, Belibi FA, He Z, Edelstein CL. Long-term rapamycin therapy in the Han: SPRD rat model of polycystic kidney disease (PKD). Nephrol Dial Transplant 2009;24(8): 2349-53. PMCID: 2727300.

8. Shillingford JM, Murcia NS, Larson CH, Low SH, Hedgepeth $\mathrm{R}$, Brown N, et al. The mTOR pathway is regulated by polycystin-1, and its inhibition reverses renal cystogenesis in polycystic kidney disease. Proc Natl Acad Sci U S A 2006; 103(14): 5466-71. PMCID: 1459378.

9. Gattone VH, 2nd, Sinders RM, Hornberger TA, Robling AG. Late progression of renal pathology and cyst enlargement is reduced by rapamycin in a mouse model of nephronophthisis. Kidney Int 2009; 76(2): 178-82.

10. Wei W, Popov V, Walocha JA, Wen J, Bello-Reuss E. Evidence of angiogenesis and microvascular regression in autosomal-dominant polycystic kidney disease kidneys: a corrosion cast study. Kidney Int 2006; 70 (7): 1261-8.

11. Liu P, Cheng H, Roberts TM, Zhao JJ. Targeting the phosphoinositide 3-kinase pathway in cancer. Nat Rev Drug Discov 2009; 8(8): 627-44.

12. Kapoor A, Figlin RA. Targeted inhibition of mammalian target of rapamycin for the treatment of advanced renal cell carcinoma. Cancer 2009; 115(16): 3618-30.
13. Perico N, Antiga L, Caroli A, Ruggenenti P, Fasolini G, Cafaro M, et al. Sirolimus therapy to halt the progression of ADPKD. J Am Soc Nephrol 2010; 21(6):1 031-40. PMCID: 2900967.

14. Serra AL, Poster D, Kistler AD, Krauer F, Raina S, Young $\mathrm{J}$, et al. Sirolimus and kidney growth in autosomal dominant polycystic kidney disease. N Engl J Med 2010; 363(9): 820-9.

15. Walz G, Budde K, Mannaa M, Nurnberger J, Wanner C, Sommerer C, et al. Everolimus in patients with autosomal dominant polycystic kidney disease. N Engl J Med 2010; 363(9): 830-40.

16. Grantham JJ, Torres VE, Chapman AB, et al. Volume progression in polycystic kidney disease. N Engl J Med 2006; 354:2122-2130.

17. Torres VE, Boletta A, Chapman A, Gattone V, Pei Y, Qian $Q$, et al. Prospects for mTOR inhibitor use in patients with polycystic kidney disease and hamartomatous diseases. Clin J Am Soc Nephrol 2010; 5(7): 1312-29.

18. Sullivan LP, Wallace DP, Grantham JJ. Chloride and fluid secretion in polycystic kidney disease. J Am Soc Nephrol 1998; 9(5): 903-16.

19. Masyuk TV, Masyuk AI, Torres VE, Harris PC, Larusso NF. Octreotide inhibits hepatic cystogenesis in a rodent model of polycystic liver disease by reducing cholangiocyte adenosine 3',5'-cyclic monophosphate. Gastroenterology 2007; 132 (3): 1104-16.

20. van Keimpema L, de Man RA, Drenth JP. Somatostatin analogues reduce liver volume in polycystic liver disease. Gut 2008; 57(9): 1338-9.

21. Ruggenenti P, Remuzzi A, Ondei P, Fasolini G, Antiga L, Ene-Iordache B, et al. Safety and efficacy of long-acting somatostatin treatment in autosomal-dominant polycystic kidney disease. Kidney Int 2005; 68(1): 206-16.

22. Caroli A, Antiga L, Cafaro M, Fasolini G, Remuzzi A, Remuzzi G, et al. Reducing polycystic liver volume in ADPKD: effects of somatostatin analogue octreotide. Clin J Am Soc Nephrol 2010; 5(5): 783-9. PMCID: 2863977.

23. van Keimpema L, Nevens F, Vanslembrouck R, van Oijen MG, Hoffmann AL, Dekker HM, et al. Lanreotide reduces the volume of polycystic liver: a randomized, doubleblind, placebo-controlled trial. Gastroenterology 2009; 137(5): 1661-8 e1-2.

24. Hogan MC, Masyuk TV, Page LJ, Kubly VJ, Bergstralh EJ, Li X, et al. Randomized clinical trial of long-acting somatostatin for autosomal dominant polycystic kidney and liver disease. J Am Soc Nephrol 2010; 21(6):1052-61. PMCID: 2900957. 